\title{
Cutaneous mucormycosis in an acute lymphoblastic leukemia child during bone marrow suppression: a case report.
}

\author{
Yalan You, Runying Zou, Xiangling $\mathrm{He}^{*}$ \\ Department of Hematology and Oncology of Children's Medical Center, Hunan Provincial People's Hospital, Changsha \\ 410005, PR China
}

\begin{abstract}
Mucormycosis is a common pathogen infecting acute lymphoblastic leukemia patients in bone marrow suppression period after chemotherapy. Infection of mucormycosis on skin is relatively rare. In this study, we present a 7-year-old boy diagnosed as acute lymphoblastic leukemia with myeloid expression and mucormycosis infection on skin. Cutaneous mucormycosis occurred on the right forearm after chemotherapy with increasing manifestations of recurrent fever, local skin necrosis and eschar, skin temperature elevation, and swelling painful feeling of right forearm. Tissue smear examination and cell culture results showed that the patient was with mucormycosis infection. Local debridement was carried out and amphotericin B applied for anti-fungal infection treatment. Although constitutional symptom was controlled after anti-infection therapy, surrounding muscles that were affected by the local necrosis kept getting worse. Therefore, free flap transplantation was undertaken along with surgical debridement, and the patient was cured with right hand function preserved completely. Follow-up examination showed that the child entered the maintain chemotherapy stage for leukemia and the local skin was growing well without infection again. In summary, our result indicated that although mucormycosis is hazardous and fatal, early detection and treatment can significantly reduce the mortality whereas drug therapy combined with surgical treatment could further enhance the cure rate.
\end{abstract}

Keywords: Cutaneous mucormycosis, Leukemia, Bone marrow suppression, Amphotericin B, Debridement. Accepted on December 28, 2017

\section{Introduction}

Patients with leukemia, especially those in the period of granulocytes deficiency during chemotherapy, have become the high-risk population of opportunistic pathogens such as fungus [1,2]. Fungus infection has no specific clinical manifestations, fungal culture positive rate is rather low, and $\mathrm{G}$ and GM experiment results are prone to be affected by various factors, therefore, early diagnosis of fungal infection is difficult [3-5]. The condition of fungus infection usually develops further, even leading to death [3-5]. Mucormycosis is one of common mycotic pathogens affecting leukemia patients in myelosuppression period. According to different infection position and infection probability, mucormycosis infections are classified into 6 types: rhinocerebral mucormycosis (39\%-75\%), pulmonary mucormycosis (24\%), disseminated mucormycosis $(23 \%)$, cutaneous mycormycosis (19\%), gastrointestinal mycormycosis, and simple central nervous system mucormycosis [6-8].

It is reported that cutaneous infection ranks the third proportion [6]. Local infection of cutaneous mucormycosis on the skin can spread easily and form disseminated infection, thus seriously threatening the life ofchildren with extremely low immunity during leukemia chemotherapy. In this study, we present the systemic antifungal and surgical treatment process in one case of cutaneous mucormycosis associated with acute lymphocytic leukemia during bone marrow suppression after chemotherapy. In addition, we investigated the clinical characteristics and therapeutic method for this infection as to provide clinical basis for early accurate diagnosis and treatment of mucormycosis.

\section{Case Presentation}

A 7-year-old boy with cough for more than half a month and fever for 5 days was admitted to Hunan Provincial People's Hospital in February 2015. The child was born in Dec 2007 while in Aug 2011, he was hospitalized with recurrent lymphadenopathy for 5 months and was diagnosed as nonHodgkin's lymphoma finally. Regular chemotherapy was carried out for more than half a year at that time and finished at Mar 2013. This time, bone marrow cytology examination showed that the child was with acute leukemia type bone marrow. Flow cytometry analysis also revealed that the patient was seized with T-cell acute lymphoblastic leukemia accompanied by myeloid antigen expression. ALL-REZ BFM 2002 chemotherapy treatment was performed since Feb 18, 2015 and the process went on smoothly. 
He was hospitalized in Nov 2015 and physical examination was performed as soon as his admition. The patient was conscious and the body temperature was $36.5^{\circ} \mathrm{C}$. No swelling of superficial lymph nodes, yellow staining on the skin, or mucous membranes was found. Other characteristics including soft neck and clear lung breath sounds could be observed without pleural friction sound. Heart rate was normal and no inner noise was detected. Abdominal examination showed the abdomen was soft without tenderness and enclosed masses. ALL-REZ BFM 2002 R1 chemotherapy was carried out regularly with dexamethasone of $20 \mathrm{mg} / \mathrm{m}^{2}$, azathioprine of $100 \mathrm{mg} / \mathrm{m}^{2}$, vincristine of $1.5 \mathrm{mg} / \mathrm{m}^{2}$, methotrexate of $1 \mathrm{~g} / \mathrm{m}^{2}$, and cytarabine of $2 \mathrm{~g} / \mathrm{m}^{2}$. At the first day of chemotherapy, lumbar puncture was conducted accompanied by intrathecal injection with $5 \mathrm{mg}$ dexamethasone, $12.5 \mathrm{mg}$ methotrexate, and $35 \mathrm{mg}$ cytarabine.

Bone marrow suppression occurred after chemotherapy and blood routine examination 6 days later showed that white blood cell was $0.37 \times 10^{9} / \mathrm{L}$, neutrophil was $0.16 \times 10^{9} / \mathrm{L}$, hemoglobin was $64 \mathrm{~g} / \mathrm{L}$, and platelet was $57 \times 10^{9} / \mathrm{L}$. All these values reduced significantly and the patient was then treated with cefoperazone sulbactam sodium to prevent infection. Recombinant human granulocyte-stimulating factor was taken to increase granulocyte at the same time. Night fever happened 1 day later and meropenem strengthen was then adopt for antiinfective therapy. Blood routine examination was performed for the second time after anti-infection and the result displayed that all blood cells including white blood cell, neutrophil, hemoglobin, and platelet remained extremely low. A red rash was discovered on the right forearm with the size of $1 \mathrm{~cm} \times 1$ $\mathrm{cm}$ and then the color of the rash developed gradually to black in the center with swelling obviously and the diameter increased to $1.5 \mathrm{~cm}$ accompanied by slightly higher skin temperature and severe pain (Figure 1).
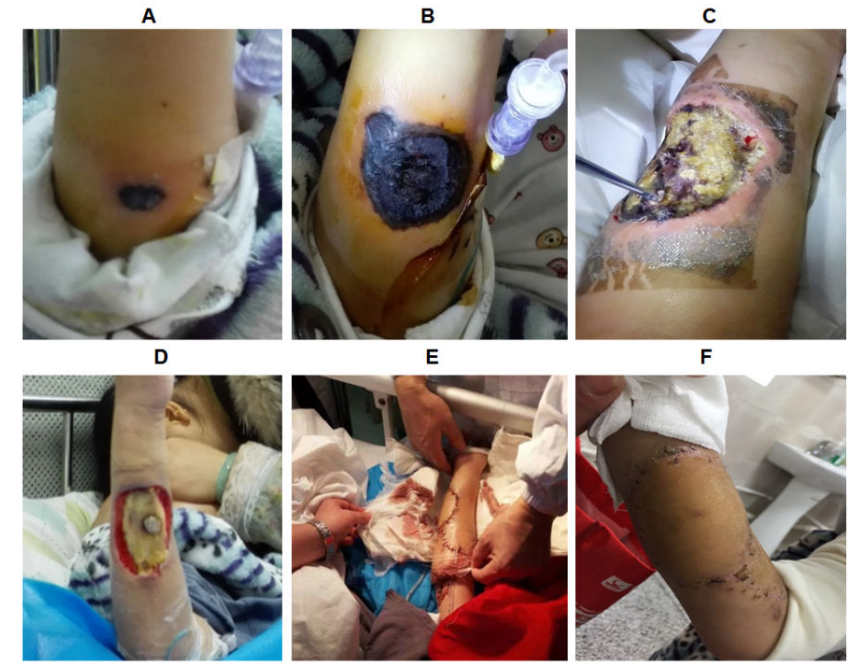

Figure 1. The local infection site of the pediatric patient. The wound skin injury size increased before operation $(A-C)$, and fresh muscle tissue was observed post-operation, with necrosis visible at the center (D).The first day after skin flap transplantation (E).Wound recovery was found after suture removal $(F)$.
The patient was with repeatedly fever and vancomycin was prescribed for anti-infection followed by platelets transfused as well as fluconazole for fungal infection prevention. C-reactive protein was detected 4 days later since vancomycin treatment, and the result showed that the value was $78.8 \mathrm{mg} / \mathrm{L}$, which was significantly higher than the normal range. Other infection related indicators were also detected and the levels of fungi (1-3)- $\beta$-D glucan and Aspergillus antigen were $64.36 \mathrm{pg} / \mathrm{ml}$ and $0.6204 \mu \mathrm{g} / \mathrm{L}$ respectively. Chest $\mathrm{CT}$ showed inflammation and a little pleural effusion in superior lobe and middle lobe of the right lung. Local necrosis on the right forearm enlarged continually with time. Smear and culture of skin tissue scarping showed visible hyphae (Figure 2), indicating the possibility of fungal infection. Therefore, amphotericin B was used with gradually increasing amount from the initial dosage of $0.1 \mathrm{mg} / \mathrm{kg}$ to strengthen the anti-fungal treatment. The dose of amphotericin B was added to the maximum amount of 25 $\mathrm{mg}(1 \mathrm{mg} / \mathrm{kg}) 3$ days after initiation. The patient's body temperature turned to normal and kept stable without fever again.

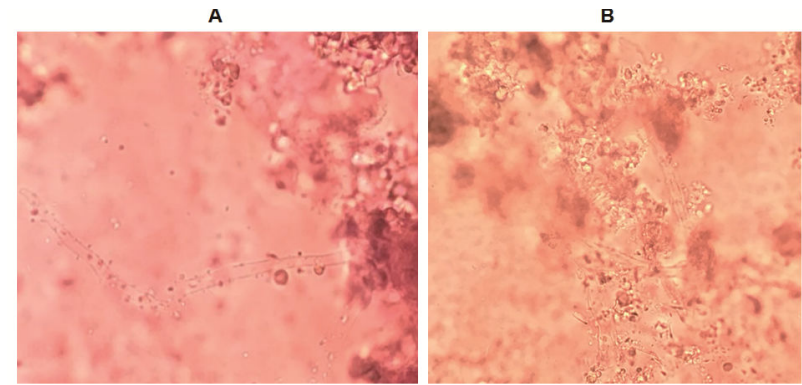

Figure 2. Tissue smear result. Wound tissues were observed under microscope at different times and fungal hypha was found.

Although general symptoms of the patient improved gradually, the region of local necrosis on the right forearm expanded continuously without control, therefore local debridement treatment was conducted subsequently. The necrosis was found to invade to as deep as muscle and even the bone surface during the debridement, hence the combination of right forearm debridement and VSD drainage was processed under the condition of general anesthesia. At the same time, the result from skin tissue culture verified the existence of mucormycosis (Figure 2). Blood routine examination was performed for the third time and the results showed that the white blood cell was $2.51 \times 10^{9} / \mathrm{L}$, the neutrophil was $1.95 \times 10^{9} / \mathrm{L}$, the lymphocyte was $0.28 \times 10^{9} / \mathrm{L}$, hemoglobin was $76 \mathrm{~g} / \mathrm{L}$, and the platelet was $47 \times 10^{9} / \mathrm{L}$, which were all slightly lower than normal range. The C-reactive protein was $9.03 \mathrm{mg} / \mathrm{L}$, slightly higher than normal range and significantly lower compared to that before surgery. VSD was removed just 1 week after operation and the edge of the wound was fresh with partial muscle tissues colored yellow (Figure 1). Debridement treatment was performed continuously and the wound surface was covered 10 days later through using circumflex scapular artery perforator flap. Amphotericin B was used successively for antifungal therapy to a total time of 46 days and the patient recovered well post operation. 


\section{Discussion}

Integral skin mucous membrane is one of the most important barriers against dermatophyte infection, especially in immunecompromised patients. Immunocompromised children with skin lesions are more prone to get fungal infection, and the condition usually aggravates and deteriorates quickly [9]. Mucormycosis is a hazardous opportunistic pathogen with high infection rate and mortality, which affects immunocompromised patients commonly in clinic. It has a strong invasiveness upon vessels and once infecting, it grows and reproduces rapidly in the infected parts and then invades the surrounding blood vessels rap (especially the arteries). The rapid growth and reproduction of mucormycosis could lead to the formation of cenobium and thrombus, which, will then block the blood vessels, resulting in vascular occlusion and ischemic tissue necrosis [10]. The tissue necrosis then gives rise to a wide range of black necrotic lesions which may cause fatal injuries to patients while vascular thrombus can further worsen the condition of tissue hypoxia and acidosis. These will in return help the proliferation and infection of mucormycosis, block the drug from the pathological tissues, and induce treatment failure $[5,11]$.

Risk factors of mucormycosis infection include diabetes, acidosis, malignancy, organ transplantation, granulocyte deficiency, glucocorticoid use, AIDS, trauma, malnutrition, and usage of deferoxamine [6,12-14]. Mucormycosis infection could also be found in patients with normal immune function, especially those with trauma or skin damage [15]. It is of great difficulty to treat mucormycosis once the infection spread to the whole body or even to the intracranial tissues, and the mortality rate would increase to as high as $80 \% \sim 90 \%$. Therefore, early diagnosis and active use of effective antifungal therapy are of great significance for the treatment of this disease. However, due to the lack of specific clinical manifestations of this disease, mucormycosis is easy to be misdiagnosed.

In this case, a small local skin lesion occurred on the right forearm of the leukemia child during bone marrow suppression period after chemotherapy. This small lesion later caused large expansion of skin necrosis. Bone marrow suppression and skin injury were the main factors of skin fungal infection. Due to the low immunity, the condition of the child progressed rapidly. The infection site kept expanded continually with systemic fever, obvious swelling and pain on the right forearm while tissue culture and smear examination indicated that the patient was with mucormycosis infection. The performance of black skin in early stage of the patient in our study were similar to that of subcutaneous hemorrhage since the platelet was extremely low at first. However, as the extent of necrosis remained enlarged after the infusion of platelets, subcutaneous bleeding was finally excluded. Definite diagnosis of mucormycosis depends on the pathological analysis and the confirmation of culture result. However, as the false negative result of culture analysis is frequently-occurring, histopathology or smear test usually becomes the only evidence for diagnosis of mucormycosis. It is reported that among the $78 \%$ patients discovered of mucormycosis infection by the histopathology or smear test, only $55 \%$ are cultureanalysis positive [6]. The morphological characteristic of mucormycosis is specific with thick hyphae $(5 \sim 15 \mathrm{~nm}$ in diameter) and irregular but rarely separated branch, which is just the opposite of Aspergillus [16,17]. In other ways, PCR method is currently a hot topic in molecular diagnosis of mucormycosis and a large amount of reports have pointed out that primer probes designed specifically to the fungal ITS, $28 \mathrm{~S}$ or other region can contribute to the detection of different fungal types. However, this method is only used in experimental research stage but not in clinical detection yet [18-21].

Currently, specifications for treatment of mucormycosis have not been developed. Most successfully treated cases are resulting from the combination of active treatment for primary disease and antifungal therapy as well as surgical operation $[15,22,23]$. It has been confirmed that amphotericin $\mathrm{B}$ is a sensitive and effective drug for mucormycosis infection treatment and this drug is widely used in clinics [5,24]. Antiinfection treatment of mucormycosis should be performed continually until the patient enters a stable condition while criterions of this stable condition include symptom remission of clinical manifestations, stability of imaging performance, and relieving of internal environment such as original immune suppression. At the same time, as the vascular occlusion and tissue necrosis caused by infection make it difficult for effective drug therapy, debridement treatment should be conducted as early as possible under an allowable physical condition. It is reported in numerous studies that surgical treatment can effectively improve the curative ratio of mucormycosis infections such as rhinocerebralmucormycosis and dermatomucormycosis $[15,22,23,25]$. The overall survival rate of mucormycosis infection is only $3 \%$ without treatment and simple amphotericin B treatment can improve this ratio to $61 \%$ while combination of amphotericin B treatment with surgical operation can further increase this rate by 1.5 times $[6,20]$.

As fungal infection always results in large area of skin damage which is difficult to recover, free skin flap coverage after amphotericin B anti-infection treatment is an excellent method for wound healing if the patient is under a good systemic condition [15]. There is also a successfully treated case reported previously about a 4-year-old child with acute lymphoblastic leukemia in induction chemotherapy combined by mucormycosis infection on the right forearm. He was cured through combination of amphotericin B therapy and surgical treatment, indicating that surgical operation is of great significance for the recovery of local mucormycosis infection. Many factors including immune function and the patient's age have important effect on the outcome for mucormycosis infection and the prognosis of children is better compared withthe elder [17,23].

In this case, the patient with mucormycosis infection during myelosuppression period was treated with antifungal therapy as well as granulocyte elevation treatment firstly. The systemic 
symptoms resulting from morrow suppression were alleviated obviously. Then combination of debridement and flap transplantation as well as other active treatment was conducted and the combination enabled great improvement of the disease. Amphotericin B was used for as long as 46 days until the skin recovered very well, followed by oral treatment of voriconazole for consolidation therapy.

\section{Conclusion}

We present here the diagnosis and treatment course of a patient with mucormycosis infection during chemotherapy. It is notable that strengthening protective measures such as avoiding skin damage and infection should be paid more attention for patients with bone marrow suppression in chemotherapy. It is also reminded that clinicians should take the infection into consideration.

\section{Acknowledgement}

This study was supported by Technology Plan Project in Hunan Science and Technology Bereau (2013FJ6028). We greatly appreciate Department of Microsurgery in Xiangya Hospital Central South University and Department of Pediatric Orthopaedics as well as Department of Microbiology in Hunan Provincial People's Hospital for active treatment and great assistance.

\section{Ethical Statement}

Prior written and informed consent were obtained from the guardian ofthis patient and the study was approved by the ethics review board of Hunan Provincial People's Hospital.

\section{References}

1. Liang L, Su LP. Etiological analysis of 67 cases of acute leukemia combined with fungal infection. Zhongguo Shi Yan Xue Ye Xue Za Zhi 2009; 17: 218-221.

2. Michel G. Fungal infections in children with malignant disease. Arch Pediatr 2011; 18: S3-S7.

3. Zheng F, Zha H, Yang D, Deng J, Zhang Z. Diagnostic values and limitations of (1,3)-beta-d-glucans and galactomannan assays for invasive fungal infection in patients admitted to pediatric intensive care unit. Mycopathologia 2017; 182: 331-338.

4. Prasad P, Fishman JA. Impact and cost of the serum galactomannan assay at a tertiary care facility. Transplantation 2014; 98: 773-780.

5. Riley TT, Muzny CA, Swiatlo E, Legendre DP. Breaking the mold: A review of mucormycosis and current pharmacological treatment options. Ann Pharmacother 2016; 50: 747-757.

6. Roden MM, Zaoutis T, Buchanan WL, Knudsen TA, Sarkisova TA, Schaufele RL, Sein M, Sein T, Chiou CC, Chu JH, Kontoyiannis DP, Walsh TJ. Epidemiology and outcome of zygomycosis: a review of 929 reported cases. Clin Infect Dis 2005; 41: 634-653.
7. Agarwal R, Kumar V, Gupta D. Pulmonary mucormycosis: two of a kind. Eur J Intern Med 2006; 17: 63-65.

8. Greenberg RN, Scott LJ, Vaughn HH, Ribes JA. Zygomycosis (mucormycosis): emerging clinical importance and new treatments. Curr Opin Infect Dis 2004; 17: 517-525.

9. Ramachandran L, Dewan S, Kumar V, Wankhade B. Mucormycosis causing pulmonary artery aneurysm. Respir Med Case Rep 2015; 16: 71-73.

10. Kauffman CA, Malani AN. Zygomycosis: an emerging fungal infection with new options for management. Curr Infect Dis Rep 2007; 9: 435-440.

11. Maertens J, Demuynck H, Verbeken EK, Zachee P, Verhoef GE, Vandenberghe P, Boogaerts MA. Mucormycosis in allogeneic bone marrow transplant recipients: report of five cases and review of the role of iron overload in the pathogenesis. Bone Marrow Transplant 1999; 24: 307-312.

12. Vaezi A, Moazeni M, Rahimi MT, de Hoog S, Badali H. Mucormycosis in Iran: a systematic review. Mycoses 2016; 59: 402-415.

13. Reinbold C, Derder M, Hivelin M, Ozil C, Al Hindi A, Lantieri L. Using free flaps for reconstruction during infections by mucormycosis: A case report and a structured review of the literature. Ann Chir Plast Esthet 2016; 61: 153-161.

14. Wu D, Zhang LM, Jiang Y. Clinical features of mucormycosis. Zhongguo Yi Xue Ke Xue Yuan Xue Bao 2010; 32: 461-464.

15. Camara-Lemarroy CR, Gonzalez-Moreno EI, RodriguezGutierrez R, Rendon-Ramirez EJ, Ayala-Cortes AS, FragaHernandez ML, Garcia-Labastida L, Galarza-Delgado DA. Clinical features and outcome of mucormycosis. Interdiscip Perspect Infect Dis 2014; 2014: 562610.

16. Salehi E, Hedayati MT, Zoll J, Rafati H, Ghasemi M, Doroudinia A, Abastabar M, Tolooe A, Snelders E, van der Lee HA, Rijs AJ, Verweij PE, Seyedmousavi S, Melchers WJ. Discrimination of aspergillosis, mucormycosis, fusariosis and scedosporiosis in formalin-fixed paraffinembedded tissue specimens using multiple real-time quantitative PCR assays. J Clin Microbiol 2016; 54: 2798-2803.

17. Ziaee A, Zia M, Bayat M, Hashemi J. Molecular Identification of Mucor and Lichtheimia species in pure cultures of Zygomycetes. Jundishapur J Microbiol 2016; 9: e35237.

18. Valle-Maldonado MI, Jácome-Galarza IE, GutiérrezCorona F, Ramírez-Díaz MI, Campos-García J, MezaCarmen V. Selection of reference genes for quantitative real time RT-PCR during dimorphism in the zygomycete Mucor circinelloides. Mol Biol Rep 2015; 42: 705-711.

19. Kasai M, Harrington SM, Francesconi A, Petraitis V, Petraitiene R, Beveridge MG, Knudsen T, Milanovich J, Cotton MP, Hughes J, Schaufele RL, Sein T, Bacher J, Murray PR, Kontoyiannis DP, Walsh TJ. Detection of a molecular biomarker for zygomycetes by quantitative PCR assays of plasma, bronchoalveolar lavage, and lung tissue 
in a rabbit model of experimental pulmonary zygomycosis. J Clin Microbiol 2008; 46: 3690-3702.

20. Riley TT, Muzny CA, Swiatlo E, Legendre DP. Breaking the mold: A review of mucormycosis and current pharmacological treatment options. Ann Pharmacother 2016; 50: 747-757.

21. Skiada A, Lanternier F, Groll AH, Pagano L, Zimmerli S, Herbrecht R, Lortholary O, Petrikkos GL, European Conference on Infections in Leukemia. Diagnosis and treatment of mucormycosis in patients with hematological malignancies: guidelines from the 3rd European Conference on Infections in Leukemia (ECIL 3). Haematologica 2013; 98: 492-504.

22. Wang SB, Li RY, Yu J. Identification and susceptibility of Rhizomucor spp. isolated from patients with cutaneous zygomycosis in China. Med Mycol 2011; 49: 799-805.

23. Spellberg B, Walsh TJ, Kontoyiannis DP, Edwards J Jr, Ibrahim AS. Recent advances in the management of mucormycosis: from bench to bedside. Clin Infect Dis 2009; 48: 1743-1751.
24. Täger FM, Zaror CL, Martínez DP. Cutaneous mucormycosis in an immunocompromised patient. Rev Chilena Infectol 2012; 29: 101-107.

25. Abuali MM, Posada R, Del Toro G, Roman E, Ramani R, Chaturvedi S, Chaturvedi V, LaBombardi VJ. Rhizomucor variabilis var. regularior and Hormographiella aspergillata infections in a leukemic bone marrow transplant recipient with refractory neutropenia. J Clin Microbiol 2009; 47: 4176-4179.

\section{*Correspondence to}

Xiangling $\mathrm{He}$

Department of Hematology and Oncology of Children's Medical Center

Hunan Provincial People's Hospital

PR China 